\title{
Spanning quadrangulations of triangulated surfaces
}

\author{
Kündgen, André; Thomassen, Carsten
}

Published in:

Universitaet Hamburg. Mathematisches Seminar. Abhandlungen

Link to article, DOI:

10.1007/s12188-016-0172-z

Publication date:

2017

Document Version

Peer reviewed version

Link back to DTU Orbit

Citation (APA):

Kündgen, A., \& Thomassen, C. (2017). Spanning quadrangulations of triangulated surfaces. Universitaet Hamburg. Mathematisches Seminar. Abhandlungen, 87(2), 357-368. https://doi.org/10.1007/s12188-016-0172-z

\section{General rights}

Copyright and moral rights for the publications made accessible in the public portal are retained by the authors and/or other copyright owners and it is a condition of accessing publications that users recognise and abide by the legal requirements associated with these rights.

- Users may download and print one copy of any publication from the public portal for the purpose of private study or research.

- You may not further distribute the material or use it for any profit-making activity or commercial gain

- You may freely distribute the URL identifying the publication in the public portal

If you believe that this document breaches copyright please contact us providing details, and we will remove access to the work immediately and investigate your claim 


\title{
Spanning quadrangulations of triangulated surfaces To the memory of Rudolf Halin.
}

\author{
André Kündgen* \\ Department of Mathematics \\ California State University San Marcos \\ San Marcos, CA 92069 \\ Carsten Thomassen ${ }^{\dagger}$ \\ Department of Applied Mathematics and Computer Science \\ Technical University of Denmark \\ DK-2800 Lyngby, Denmark
}

March 28, 2015

\begin{abstract}
In this paper we study alternating cycles in graphs embedded in a surface. We observe that 4-vertex-colorability of a triangulation on a surface can be expressed in terms of spanninq quadrangulations, and we establish connections between spanning quadrangulations and cycles in the dual graph which are noncontractible and alternating with respect to a perfect matching. We show that the dual graph of an Eulerian triangulation of an orientable surface other than the sphere has a perfect matching $M$ and an $M$-alternating noncontractible cycle. As a consequence, every Eulerian triangulation of the torus has a nonbipartite spanning quadrangulation. For an Eulerian triangulation $G$ of the projective plane the situation is different: If the dual graph $G^{*}$ is nonbipartite, then $G^{*}$ has no noncontractible alternating cycle, and all spanning quadrangulations of $G$ are bipartite. If the dual graph $G^{*}$ is bipartite, then it has a noncontractible, $M$-alternating cycle for some (and hence any) perfect matching, $G$ has a bipartite spanning quadrangulation and also a nonbipartite spanning quadrangulation.
\end{abstract}

Keywords: Vertex-coloring, edge-coloring, quadrangulation, alternating cycle. Mathematics Subject Classification:05C10, 05C15

\section{Introduction}

5-vertex-colorability of a graph on a fixed surface is well understood in the following sense: There are only finitely many minimal graphs that are not properly 5-vertex-colorable, [9]. There is no analogous result for 4-vertex-coloring (except when the surface is the sphere) because of the Ballentine-Fisk triangulations, see [6], page 241.

Tait proved in 1880 (see Jensen and Toft [3] page 16) that there is a one-to-one correspondance between the 4-vertex-colorings of a planar triangulation and the proper 3-edge-colorings of the dual

\footnotetext{
${ }^{*}$ This work was done while the first author visited the Technical University of Denmark supported in part by the Danish Natural Science Research Council grant number 09-072584.

${ }^{\dagger}$ Research partly supported by ERC Advanced Grant GRACOL.
} 
graph. A proper 3-edge coloring of the dual graph can also be expressed as an edge-coloring of the triangulation such that every triangle receives all three colors, or equivalently, the union of any two colors is a spanning quadrangulation. With a slight modification, this extends to higher surfaces as follows:

Proposition 1 A triangulation $G$ of a surface has a proper 4-vertex-coloring if and only if its edge set has a proper 3-edge-coloring such that any two colors induce a bipartite spanning quadrangulation.

The "if" part is trivial as the union of two bipartite graphs is 4-vertex-colorable. To prove the "only if" part, we consider a 4 -vertex-coloring where we think of the four colors as the ordered pairs $(i, j)$ where each of $i, j$ is 0 or 1 . We give each edge of the triangulation the color which is the sum of the colors of the ends, where addition is taken modulo 2 . Then the edge set is colored with the colors $(0,1),(1,0),(1,1)$, and any two color classes form a spanning quadrangulation $Q$. To see that $Q$ is bipartite, let us assume that $C$ is an odd cycle in $Q$. The sum of colors of the edges in $C$ is the sum of colors of vertices where each vertex appears twice. That is, the sum is $(0,0)$. As the edges of $Q$ have only two colors, one of the colors appears an even number of times with total sum $(0,0)$, and the other color appears an odd number of times with total sum $\neq(0,0)$, a contradiction.

Ramamurthi et al. [4] asked if every graph of large width on a fixed surface has a 2-vertexcoloring such that no face boundary is monochromatic. It suffices to verify this for triangulations. If we delete the edges of such a 2-vertex-colored triangulation joining the vertices of the same color we get a bipartite spanning quadrangulation. If this question can be answered affirmatively, then one may drop the condition of the width and conjecture that, for each surface, there are only finitely many minimal graphs with no 2 -vertex-coloring satisfying the condition that no face boundary is monochromatic. This might be an intermediate step towards gaining a better understanding of 4 -vertex-colorability of graphs on a fixed surface.

The edges in the triangulation but outside of the spanning quadrangulation form a perfect matching in the dual graph. The purpose of this paper is to establish connections between matchings and their alternating cycles in the dual graph of a triangulation (see Section 3 for a definition) and then apply these connections to study spanning quadrangulations. We apply these results in particular to triangulations on the torus or the projective plane as explained in the abstract. For example, it follows that the above 2-vertex-coloring exists for every Eulerian triangulation of the projective plane, even without the width condition. On the torus the width condition is needed even for Eulerian triangulations as shown by $K_{7}$ on the torus. More generally, if $K_{n}$ triangulates a surface other than the sphere, then every spanning quadrangulation is non-bipartite (except when $n=4$ ). To see this we use Euler's formula $n-e+f=2-g$ (where $g \geq 1$ is the Euler genus) to conclude that $K_{n}$ has $f \leq n(n-1) / 2-n+1$ faces and that the number of edges in a spanning quadrangulation is $n(n-1) / 2-f / 2>n^{2} / 4$ so the quadrangulation contains a triangle, by Turán's theorem.

\section{Notation and terminology}

We follow the notation and terminology of $[1,6]$.

Let $G$ be a graph embedded in a surface $\Sigma$ with large width, where the (face) width or representativity is the minimum number of times a noncontractible curve intersects the drawing of $G$.

We are interested in 2-coloring the vertices of $G$ such that no face is monochromatic. Observe that we can extend $G$ to a triangulation by adding edges without reducing its width, and this only 
makes 2-coloring $G$ harder. This may create double edges (and $G$ may have had such edges to begin with,) but such double edges would form a contractible digon, that splits off a planar piece which can easily be 2-colored. Thus we can identify the two digon edges, and we can assume that $G$ is simple.

Throughout this paper we assume that $G$ is a (simple) triangulation, and a spanning quadrangulation $Q$ refers to a spanning subgraph of $G$ such that every face is bounded by a cycle on 4 vertices. Observe now that $G$ can be 2 -colored such that no face is monochromatic iff $G$ contains a spanning quadrangulation $Q$ which is bipartite. Indeed, a proper 2-coloring of $Q$ immediately yields the 2-coloring for $G$, since the three vertices of a face of $G$ are all contained in the same 4-gon face of $Q$. Conversely, removing all the edges whose endpoints have the same color in a 2-coloring of $G$, we obtain a bipartite graph $Q$, and since every face has two colors it is easy to see that the faces of $Q$ will be 4-gons.

Next we observe that every spanning quadrangulation $Q$ of $G$ corresponds to a perfect matching $M$ in the dual graph $G^{*}$, namely the set of edges that is dual to the edges in $E(G)-E(Q)$. Since $G^{*}$ is 3-regular and 2-connected, it follows from Petersen's theorem that $G^{*}$ must have a perfect matching. Thus every triangulation $G$ has a spanning quadrangulation $Q$, but $Q$ may or may not be bipartite.

Definition 2 If $G$ is a triangulation such that every spanning quadrangulation of $G$ is bipartite (respectively nonbipartite), then $G$ is called q-even (respectively q-odd.) If $G$ has spanning quadrangulations of both kinds, then it is q-mixed.

It seems like an interesting question in its own right to categorize the triangulations according to their $q$-parity. The question from [4] we started out with can now be restated as follows:

Question 3 Are there q-odd triangulations of high width?

Hutchinson, Richter and Seymour [2] proved that an Eulerian triangulation of an orientable surface with high width must be 4 -colorable (and thus combining 2 colors, they are 2-colorable such that no face is monochromatic.) Hence it cannot be $q$-odd.

\section{Alternating cycles and spanning quadrangulations}

The purpose of this section is to study the interplay between spanning quadrangulations of a triangulation, and matchings and their alternating cycles in the dual graph.

We start our investigation with the observation that if we have a planar graph in which at most one face has odd length, then in fact every face has even length and the graph is bipartite. It follows immediately that every triangulation of the plane is $q$-even. The same argument shows that in fact every surface separating cycle of a spanning quadrangulation of an arbitrary surface must be of even length. Thus a spanning quadrangulation is nonbipartite if and only if it contains a surface nonseparating (in particular noncontractible) odd cycle.

The following notion seems to be useful when going between bipartite and nonbipartite spanning quadrangulations. Given the perfect matching $M$ in $G^{*}$ that corresponds to the spanning quadrangulation $Q$, we say that a cycle $C$ in $G^{*}$ is $M$-alternating if it alternates between edges in $M$ and edges not in $M$. The symmetric difference $E(C) \Delta M$ is another perfect matching in $G^{*}$ corresponding to a new spanning quadrangulation $Q_{C}$ of $G$. If $C_{1}, C_{2}, \ldots, C_{k}$ are pairwise disjoint $M$-alternating cycles, then we define recursively $Q_{C_{1} C_{2} \ldots C_{k}}=\left(Q_{C_{1} C_{2} \ldots C_{k-1}}\right)_{C_{k}}$. 
Lemma 4 Let $Q$ be a spanning quadrangulation of a triangulation $G$, with corresponding matching $M$ in $G^{*}$ and $M$-alternating cycle $C$ in $G^{*}$ and let $H=Q-E(C)^{*}=Q_{C}-E(C)^{*}$.

1. $Q$ and $Q_{C}$ are both nonbipartite if and only if $H$ is nonbipartite.

2. $Q$ and $Q_{C}$ are both bipartite if and only if $H$ is bipartite and $C$ is surface separating.

3. Exactly one of $Q$ and $Q_{C}$ is bipartite if and only if $H$ is bipartite and $C$ surface nonseparating.

Proof. It suffices to prove the backward direction for all three statements, and this is obvious for the first statement. So now we may assume that $H$ is bipartite. Observe that if $C$ meets a face $u v w$ in $G$, say with $u v$ in $Q$ and $v w$ in $Q_{C}$, then $u w$ must be in $H$. Suppose first that $C$ is surface nonseparating. Then any curve from $u$ to $v$ in the surface that does not meet $C$ and meets the drawing only in $V(G)$ yields a $u v$-path in $G-E(C)^{*}$, which in turn yields a $u v$-path in $H$, so that $H$ is connected. In this case the 2-coloring of $H$ is unique. In this 2-coloring $u, w$ receive different colors, and we may assume that $v$ receives the same color as say $w$. Going along $C$ we see that all edges in $E(C)^{*} \cap M^{*}$ are properly colored, whereas all edges in $E(C)^{*}-M^{*}$ are monochromatic, so that $Q$ is bipartite, but $Q_{C}$ is not.

If $C$ is surface separating, then $H$ has two components $H_{1}$ (containing some $u, w$ as above) and $H_{2}$ (containing $v$.) By the same argument as before the 2-coloring of $H$ in which the colors of $v$ and $w$ agree shows that $Q$ is bipartite, whereas the 2-coloring of $H$ in which the colors of $v$ and $u$ agree shows that $Q_{C}$ is bipartite.

Observe that the condition that $C$ is surface separating is equivalent to the fact that $H$ is disconnected. This lemma immediately implies

Proposition 5 The following are equivalent for a triangulation $G$ of any surface.

1. $G$ is q-mixed.

2. $G$ has a nonbipartite spanning quadrangulation, and for some (and hence every) nonbipartite spanning quadrangulation $Q$ with associated matching $M$ in $G^{*}$ there is a collection of vertexdisjoint surface nonseparating $M$-alterning cycles $C_{1}, C_{2}, \ldots, C_{k}$ in $G^{*}$ such that $Q_{C_{1} C_{2} \ldots C_{k}}$ is a bipartite spanning quadrangulation.

Proof. It is obvious that 2 implies 1 . To see that 1 implies 2 let $Q$ be a nonbipartite spanning quadrangulation, and $Q^{\prime}$ be a bipartite one. The symmetric difference of their corresponding matchings $M \Delta M^{\prime}$ is a collection of cycles $C_{1}, \ldots, C_{m}$ that are alternating for both matchings. Observe that $Q=Q_{C_{1} C_{2} \ldots C_{m}}^{\prime}$, so that by Lemma 4.3 some of these cycles must be surface nonseparating, say $C_{1}, \ldots, C_{k}$. Now observe that $Q_{C_{1} \ldots C_{k}}=Q_{C_{k+1} \ldots C_{m}}^{\prime}$, and the latter is bipartite by Lemma 4.2 .

Another easy consequence of Lemma 4.3 is the following.

Corollary 6 If $G$ is a triangulation of the projective plane with a spanning quadrangulation $Q$ that has a surface nonseparating $M$-alternating cycle $C$ in $G^{*}$, then $G$ is q-mixed.

Proof. Observe that since $C$ is a nonseparating cyle in the projective plane, $Q-E(C)^{*}$ is a planar graph such that at most one face is not a 4-gon, and thus $Q-E(C)^{*}$ is bipartite.

Inserting a vertex into each face of a spanning quadrangulation we obtain an Eulerian triangulation. Eulerian triangulations have nice properties. For example Hutchinson, Richter and Seymour 
[2] proved that an Eulerian triangulation of an orientable surface with high width must be 4colorable (and thus combining 2 colors, they are 2-colorable such that no face is monochromatic.) The same statement is not true for nonorientable surfaces, since there are Eulerian triangulations of large width that are not 4-colorable. The standard construction, given in the next result, turns out to be $q$-mixed in any surface, orientable or not.

Proposition 7 Every surface $\Sigma$ other than the plane has an Eulerian q-mixed triangulation of arbitrarily high width.

Proof. Start with a nonbipartite quadrangulation $Q^{\prime}$ of $\Sigma$ with high width. (To see that these exist one can start from a toroidal embedding of the cartesian product of two very long odd cycles $C_{2 k+1}$, and then increase the genus by successively deleting the interior of some pairs of $m \times m$ grids and then pairwise identifying the boundaries. As long as $m$ is large enough and these grids are mutually far enough apart the resulting spanning quadrangulation will be of high width, and nonbipartite as long as some copy of $C_{2 k+1}$ is unaffected. This construction works when $\Sigma$ is distinct from the nonorientable surfaces with one, two, or three crosscaps. For those nonorientable surfaces we can instead start with a quadrangulation of the sphere that contains a suffciently large grid, remove an $m \times m$-grid for each desired crosscap, and make each vertex on the boundary of such a removed grid adjacent to the opposite vertex on this boundary.)

To each face $f$ of $Q^{\prime}$ we add a new vertex $v_{f}$ to $Q^{\prime}$ and make it adjacent to every vertex in $f$ to obtain a new graph $G$. $G$ is clearly an Eulerian triangulation. Moreover $G$ contains a nonbipartite spanning quadrangulation obtained from $Q^{\prime}$ by adding in the vertices $v_{f}$ together with two edges each to nonadjacent vertices of $f$. But $G$ also contains a bipartite spanning quadrangulation, which can be obtained by removing the edges of $Q^{\prime}$ from $G$, since then the vertices of $Q^{\prime}$ form one color class, and the facial vertices $v_{f}$ form the other color class.

\section{The existence of noncontractible alternating cycles in bipartite graphs}

In the previous section we have tried to show how to make use of noncontractible alternating cycles if they exist. We now address the question of existence. For bipartite graphs the existence can be established by cycle space methods.

We say that a cycle in a graph $G$ is alternating if it is $M$-alternating for some perfect matching $M$. Let $Z(G)$ denote the cycle space of $G$ (see [1] page 35 for a definition), and let $Z^{\prime}(G)$ be the subspace of $Z(G)$ that is generated by the alternating cycles.

Proposition 8 If $M$ is a fixed perfect matching in a graph $G$, then $Z^{\prime}(G)$ is generated by the $M$-alternating cycles.

Proof. It suffices to prove that every alternating cycle $C$ is the modulo 2 sum (i.e. the symmetric difference) of $M$-alternating cycles. As $C$ is alternating, there are two perfect matchings $M_{1}, M_{2}$ such that the edges in $C$ are alternately from $M_{1}$ and $M_{2}$. We may assume that $M_{1}, M_{2}$ agree outside of $C$. We now consider the modulo 2 sum of all cycles in $M \cup M_{1}$ and all cycles in $M \cup M_{2}$. This sum equals $C$.

Theorem 9 If $G$ is a bipartite graph in which every edge is contained in some perfect matching, then $Z^{\prime}(G)=Z(G)$. 
Proof. It suffices to consider the case where $G$ is connected. Let $A, B$ be the bipartition of $G$. As $G$ is connected and every edge is in a perfect matching, there must be strict inequality in Hall's condition for every proper subset of $A$. (For, if $S$ is a proper subset of $A$ and the set $N(S)$ of neighbors of $S$ has the same cardinality as $S$, then there is an edge from $A \backslash S$ to $N(S)$ (because $G$ is connected) but no such edge is in a perfect matching.) So, if $u, v$ are vertices in $A, B$, respectively, then $G-u-v$ has a perfect matching $M^{\prime}$. As also $G$ has a perfect matching, $G$ has an $M^{\prime}$-alternating path from $u$ to $v$.

Let $H$ be a maximal connected subgraph of $G$ with the following properties:

(i) Each edge of $H$ is in a perfect matching of $H$.

(ii) $G-V(H)$ is empty or has a perfect matching $M$.

(iii) $Z^{\prime}(H)=Z(H)$.

As every alternating cycle satisfies the conditions (i),(ii),(iii), $H$ is nonempty. We claim that $H=G$. So assume (reductio ad absurdum) that $G$ has an edge $e$ which is not in $H$, but is incident with a vertex $u$ in $H$. As $e$ is in a perfect matching of $G, G$ has an $M$-alternating path $P$ from $u$ to some $v \in V(H)$, which starts with $e$ and which has no other vertex in common with $H$. Also, $H-u-v$ has a perfect matching $M^{\prime}$ such that $H$ has an $M^{\prime}$-alternating path from $P^{\prime}$ from $u$ yo $v$. Now $P \cup P^{\prime}$ is an alternating cycle in both $G$ and $H^{\prime}=H \cup P$. Now $Z^{\prime}(H)=Z(H)$, and the dimension of $Z\left(H^{\prime}\right)$ exceeds the dimension of $Z(H)$ by precisely 1 . The dimension of $Z^{\prime}\left(H^{\prime}\right)$ is strictly greater than the dimension of the cycle space of $H$, and thus $Z^{\prime}\left(H^{\prime}\right)=Z\left(H^{\prime}\right)$. Therefore $H^{\prime}$ satisfies (i),(ii),(iii), a contradiction.

Observe that every edge that is in an alternating cycle must be in a perfect matching, so that the second condition in Theorem 9 is essentially necessary. Moreover, the conclusion in Theorem 9 does not hold for nonbipartite graphs because (modulo 2 sums of) alternating cycles have an even number of edges. It would be useful if the alternating cycles (in a connected graph where every edge is in a perfect matching) would generate the subspace $Z^{\prime \prime}(G)$ of $Z(G)$ consisting of all eulerian subgraphs with an even number of elements. (The dimension of $Z^{\prime \prime}(G)$ is one less than the dimension of $Z(G)$ when $G$ contains an odd cycle.) But the following example shows that the alternating cycles may generate a subspace of much smaller dimension: For $k \geq 2$ take a cycle $v_{1} v_{2} \ldots v_{4 k} v_{1}$, and add all edges $v_{4 i} v_{4 i-2}, v_{4 i-1} v_{4 i-3}, i=1,2, \ldots, k$. For this graph $G$ the dimension of $Z(G)$ is $2 k+1$ whereas $Z^{\prime}(G)$ has dimension $k+1$.

Theorem 10 Let $H$ be a cubic bipartite graph drawn on any surface. Let $M$ be any perfect matching of $H$.

If $H$ has a noncontractible cycle, then $H$ has an $M$-alternating noncontractible cycle.

If $H$ has a onesided cycle, then $H$ has an $M$-alternating onesided cycle.

If $H$ has a surface nonseparating cycle, then $H$ has an $M$-alternating surface nonseparating cycle.

Proof. Observe that the edge set of a cubic bipartite graph can be decomposed into perfect matchings, and so each edge is in a perfect matching. Thus the last two statements follow directly from Proposition 8 and Theorem 9 because a surface nonseparating cycle cannot be a modulo 2 sum of surface separating cycles, and a onesided cycle cannot be a modulo 2 sum of twosided cycles. The former obervation follows from the fact that a cycle is surface separating if and only if every closed curve that avoids $V(H)$ crosses its edges an even number of times, and this property is preserved under modulo 2 sum. The latter observation can be derived similarly from the fact that a cycle is onesided if and only if it has an odd number of edges with negative signature, see [6] page 101.

It is possible that a noncontractible cycle $C$ is a modulo 2 sum of contractible cycles so this case needs a different argument. We need only consider the case where $C$ divides the surface into 
two surfaces, each distinct from the sphere. If $H$ is a 2-cell embedding of one of these, then the part of $H$ in that surface has a surface nonseparating cycle (see [6] page 106) and we are done. Otherwise each of the two parts of the surface has a face which is not homeomorphic to a disc. We replace each of these faces by a tube whose boundaries are the two face boundaries. In this new surface $C$ is surface nonseparating. Hence $H$ has an $M$-alternating surface nonseparating cycle $C^{\prime}$ in this new surface. Then $C^{\prime}$ is noncontractible in the original surface.

\section{$5 \quad$ Eulerian triangulations of the projective plane}

Theorem 11 Let $G$ be an Eulerian triangulation of the projective plane.

If $G^{*}$ is bipartite, then $G$ is q-mixed.

If $G^{*}$ is not bipartite, then $G$ is q-even.

Proof. Observe that $G^{*}$ must have a surface nonseparating cycle (see [6] page 106). Thus the first statement follows from Theorem 10 with $H=G^{*}$ and Corollary 6 and it suffices to consider the case when $G^{*}$ is not bipartite.

Observe that if $G^{*}$ contains any noncontractible even cycle $C^{*}$, then $G^{*}$ is bipartite. This follows since if we cut the projective plane along $C^{*}, G^{*}$ yields a graph embedded in the plane whose exterior face is bounded by a cycle $C$ of length $2\left|C^{*}\right|$. Since $G$ is Eulerian all faces of $G^{*}$ are even length, and this graph is properly 2-colorable. This coloring yields a proper 2-coloring of $G^{*}$ since $\left|C^{*}\right|$ being even implies that two copies of the same vertex of $G^{*}$ on $C$ receive the same color. Thus we may now assume that every noncontractible cycle in $G^{*}$ is odd.

Now suppose that $G$ contains a nonbipartite spanning quadrangulation $Q$, and let $C$ be an odd cycle in $Q$. Then $C$ must be noncontractible, since otherwise its interior forms a planar bipartite graph, contradicting the fact that $C$ has odd length. Let $E^{*}$ be the set of edges in $G^{*}$ that are dual to the edges in $C$. Removing the edges in $E^{*}$ from $G^{*}$ we obtain a planar graph $H$ with at most one face of odd length (because $C$ is surface nonseparating and hence $G^{*}-V(C)$ is connected) which must therefore be bipartite with partition $V_{0} \cup V_{1}$. Since the perfect matching in $G^{*}$ that corresponds to the edges of $E(G)-E(Q)$ is still a perfect matching in $H$, we obtain that $\left|V_{0}\right|=\left|V_{1}\right|$.

Consider any edge $u v \in E^{*}$. Observe that there is a noncontractible cycle in $G^{*}$ that meets $E^{*}$ only in the edge $u v$. Since this cycle is odd it follows that $u, v$ are in the same part $V_{i}$. Thus an edge in $G^{*}$ has endpoints in both $V_{0}, V_{1}$ if and only if it is not in $E^{*}$. Let $e_{i}$ denotes the number of edges induced by $V_{i}$. Since $G^{*}$ is cubic counting the number of edges between $V_{0}$ and $V_{1}$ yields $3\left|V_{0}\right|-2 e_{0}=3\left|V_{1}\right|-2 e_{1}$. Thus $e_{0}=e_{1}$, contradicting the fact that $e_{0}+e_{1}$ is odd, since it counts the number of edges in $C$.

A triangle replacement of a triangulation $G$ on a surface is the triangulation obtained by subdividing each edge of $G$ and pairwise connecting the three new vertices of any face. By doing sufficiently many triangle replacements of $G$ we obtain a graph $G^{\prime}$ whose width is strictly larger than the width of $G$. Moreover $G^{\prime}$ is Eulerian if and only if $G$ is, and the parity of a cycle in $G^{*}$ is the same as that of the corresponding cycle in $G^{*}$.

Corollary 12 (i) There are no q-odd Eulerian triangulations of the projective plane.

(ii) There are q-mixed Eulerian triangulations of the projective plane of arbitrarily large width.

(iii) There are q-even Eulerian triangulations of the projective plane of arbitrarily large width.

Proof. Theorem 11 implies (i). 
Proposition 7 implies (ii).

To prove (iii), let $G_{0}$ be any nonbipartite Eulerian triangulation of the projective plane. Thus if we successively let $G_{i+1}=G_{i}^{\prime}$, then we obtain Eulerian triangulations of arbitrarily large width whose dual has a noncontractible odd cycle.

We also get the following result:

Corollary 13 Let $G$ be an Eulerian triangulation of the projective plane such that $G^{*}$ is nonbipartite. Then $G$ is 4-vertex-colorable if and only if $G^{*}$ is 3-edge-colorable.

Proof. As pointed out in the introduction, if $G$ is 4 -vertex colorable, then $G^{*}$ is 3-edgecolorable. If $G^{*}$ is 3-edge-colored, then, by Theorem 11, the corresponding three spanning quadrangulations of $G$ are all bipartite. Thus $G$ is 4-vertex-colorable.

Eulerian triangulations of the projective plane may be 5-chromatic. Mohar [5] characterized these. Using that characterization we get:

Corollary 14 Let $G$ be a 5-chromatic Eulerian triangulation of the projective plane. Then $G^{*}$ is bipartite.

Proof. Mohar [5] proved that $G$ contains a nonbipartite quadrangulation $Q$ which is not necessarily spanning. Consider now a face of $Q$ and the part $H$ of $G^{*}$ which is inside that face. Since $G$ is Eulerian, $H$ is bipartite and we can assume that either $H=K_{2}$ or all its vertices have degree 3 , except for four vertices of degree 2 so that no three of these four vertices have the same color. Now Hall's theorem implies that $H$ has a perfect matching. Using these matchings in each face of $Q$ we get a (now spanning) quadrangulation of $G$ which contains all edges of $Q$ and which is therefore nonbipartite. So $G$ is not $q$-even, and it follows from Theorem 11 that it is in fact $q$-mixed, and that $G^{*}$ is bipartite.

Since cubic bipartite graphs are 3-edge-colorable, the two previous corollaries yield

Corollary 15 Let $G$ be an Eulerian triangulation of the projective plane. Then $G^{*}$ is 3 -edgecolorable.

\section{The existence of noncontractible alternating cycles in orientable surfaces}

Theorem 16 If $H$ is a cubic graph drawn in an orientable surface $\Sigma$ such that each face is bounded by a cycle of even length and such that $H$ has at least one noncontractible cycle, then $H$ has a perfect matching $M$ and a noncontractible $M$-alternating cycle $C$.

Proof. Since every face of $H$ is bounded by a cycle, $H$ is 2-connected. Since $H$ is cubic and 2-connected it has, for each edge $e$, a perfect matching containing $e$. Suppose (reductio ad absurdum) that, for every perfect matching $M$ of $H$, every $M$-alternating cycle $C$ is contractible. Pick $M$ and $C$ such that the interior of $C$ contains the maximum number of edges. For each vertex $v$ let $v v^{\prime}$ denote the edge in $M$ containing it. Since $H$ is connected and not entirely in the interior of $C$, there must be an edge $u v$ in the exterior of $C$ with $v \in V(C)$. Observe that the edge $v v^{\prime}$ is in $C$, and thus $u v \notin M$. 
If $M^{\prime}$ is any perfect matching of $H$ that contains $u v$, then the symmetric difference $M \Delta M^{\prime}$ contains an $M$-alternating cycle $v_{1} v_{2}, \ldots v_{2 k} v_{1}$ through $u v$ and $v v^{\prime}$, where $v_{1}=v, v_{2}=u$. If we let $i$ be the smallest index with $i>1$ such that $v_{i} \in V(C)$, then $v_{i} v_{i+1}$ is in $M \cap E(C)$, and thus $i$ is even. Now if both $v^{\prime}=v_{2 k}$ and $v_{i}^{\prime}=v_{i+1}$ are on the same $v, v_{i}$-segment of $C$, then we obtain an $M$-alternating cycle $C^{\prime}$ by combining this $M$-alternating segment with $v_{i} \ldots v_{1}$. If $C^{\prime}$ is noncontractible, then we are done. If $C^{\prime}$ is contractible, then by the 3-path property (see [6], page 110) $C \Delta C^{\prime}$ is also a contractible cycle, and either $C^{\prime}$ or $C \Delta C^{\prime}$ has an interior which is strictly larger than that of $C$. Moreover, $C \Delta C^{\prime}$ is an alternating cycle for the matching $M \Delta C$, contradicting our choice of $M$ and $C$.

Thus we may assume that if an $M$-alternating path has endpoints $u, v \in V(C)$, but all edges and other vertices outside of $C$, then $u^{\prime}, v^{\prime}$ must be in different $u, v$-segments of $C$.

Put $C=u_{1} u_{2} \ldots u_{m} u_{1}$ where $m$ is even.

We say that a path $P$ is a $C$-detour if $P$ is a path from a vertex $u_{i}$ in $C$ to a vertex $u_{j}$ in $C$ such that each intermediate vertex (if any) is not in $C$. If $P$ is in $\operatorname{Int}(C)$, then $P$ is an internal $C$-detour. (We will use int $(C)$ to denote the set of all vertices and edges of $G$ that meet the interior of $C$ whereas $\operatorname{Int}(C)$ is the subgraph of $G$ consisting of all the vertices and edges that are in $\operatorname{int}(C)$ or $C$.) Otherwise, $P$ is an external $C$-detour. If a $C$-detour $P$ is $M$-alternating, and $u_{i}^{\prime}=u_{i+1}$ and $u_{j}^{\prime}=u_{j+1}$, then we say that $P$ is positive. If $u_{i}^{\prime}=u_{i-1}$ and $u_{j}^{\prime}=u_{j-1}$, then we say that $P$ is negative. We have just proved that

Claim 1: Every $M$-alternating external $C$-detour is either positive or negative.

\section{Moreover:}

Claim 2: If $P$ is an $M$-alternating external $C$-detour and $P^{\prime}$ is a subpath of $C$ such that $P \cup P^{\prime}$ is a cycle, then $P \cup P^{\prime}$ has odd length and is therefore surface nonseparating.

Proof of Claim 2: Since every face has even length, it follows that every surface separating cycle has even length, since otherwise after separating we would arrive at a graph with exactly one face of odd length, but then its dual would have precisely one vertex of odd degree.

Two disjoint $C$-detours overlap if their union with $C$ form a subdivision of $K_{4}$. (For the more general definition of $C$-bridges (also sometimes called $C$-components) and overlapping $C$-bridges, see e.g. [8] or [6], page 7.)

Claim 3: A positive $M$-alternating external $C$-detour cannot overlap a negative $M$-alternating external $C$-detour.

Proof of Claim 3: For otherwise, the union of the two $M$-alternating $C$-detours and two segments of $C$ would form an $M$-alternating cycle $C^{\prime}$. Since $\Sigma$ is orientable it is easy to see that the interior of $C$ contains a curve which has only its ends in common with $C^{\prime}$ and which hits $C^{\prime}$ on the right hand side and the left hand side, respectively. Therefore $C^{\prime}$ is surface nonseparating and hence noncontractible. This proves Claim 3.

Now let $C^{\prime}$ be an $M$-alternating cycle containing an edge which is incident with $C$ but in the exterior of $C$. Then $C$ can be divided into segments $P_{1}, P_{1}^{\prime}, P_{2}, P_{2}^{\prime}, \ldots$ where $P_{i}$ is an $M$-alternating external $C$-detour and $P_{i}^{\prime}$ is a path in $\operatorname{Int}(C)$ for $i=1,2, \ldots$ As $\operatorname{Int}(C)$ is bipartite, we get

Claim 4: For each $i=1,2, \ldots, P_{i}$ and $P_{i+1}$ have different signs.

In particular, $C^{\prime}$ has at least two $M$-alternating external $C$-detours.

Assume that $C^{\prime}$ is chosen such that it has as few $M$-alternating external $C$-detours as possible. Subject to that we assume that $C^{\prime}$ has as few $M$-alternating internal $C$-detours as possible 
Claim 5: $C^{\prime}$ has precisely two $M$-alternating external $C$-detours.

Proof of Claim 5: Suppose (reductio ad absurdum) that $C^{\prime}$ has at least three (and hence at least four) external $M$-alternating $C$-detours.

We choose the notation so that $P_{1}$ is a path $u_{2} \ldots u_{a}$ with $u_{2}^{\prime}=u_{1}, u_{a}^{\prime}=u_{a-1}$ such that $a$ is minimal. Now considering $P_{2}: u_{b} \ldots u_{c}$, it follows from Claim 4 that $u_{b}^{\prime}=u_{b+1}, u_{c}^{\prime}=u_{c+1}$. The minimality of $a$ and Claim 3 imply that $b>a$ and $c>a$. Thus $P_{1}^{\prime}$ contains an internal $C$-detour $P_{1}^{\prime \prime}$ joining a vertex $u_{\alpha}$ with a vertex $u_{\beta}$ such that $2<\alpha<a<\beta$. (Note that $P_{1}^{\prime}$ may not be a $C$-detour. $P_{1}^{\prime}$ is the union of internal $C$-detours and segments of $C$.) Now we choose an internal $C$-detour $Q$ joining a vertex $u_{\alpha}$ with a vertex $u_{\beta}$ (with $\beta>\alpha$ ) such that $\beta-\alpha$ is minimum, where we do not necessarily require $\alpha<a<\beta$.

$Q$ divides $C$ into two paths $Q_{1}: u_{\alpha+1} \ldots u_{\beta-1}$ and $Q_{2}: u_{\beta+1} \ldots u_{\alpha-1}$. Observe that no external $C$-detour $P_{i}: u_{b} \ldots u_{c}$ can have both endpoints in $Q_{1}$. Indeed, suppose that $\alpha<b<c<\beta$ and $u_{c}^{\prime}=u_{c-1}$ with the other cases being similar: by the minimality of $Q, P_{i+1}: u_{d} \ldots u_{e}$ must have $b<d<c$ and Claim 3 and 4 now imply that $b<e<d$. Continuing in this matter the intervals $[b, c],[e, d] \ldots$ corresponding to $P_{i}, P_{i+1}, \ldots$ form a nested sequence, but this is impossible, since it leaves no way for $C^{\prime}$ to connect back to $u_{1}$.

Case 1: $u_{\alpha}^{\prime}=u_{\alpha+1}$.

Since $\operatorname{Int}(C)$ is planar and bipartite, it follows that $u_{\beta}^{\prime}=u_{\beta-1}$. Now traverse $C^{\prime}$ starting with $u_{\alpha} u_{\alpha+1}$ until we meet a $C$-detour. The minimality of $Q$ ensures that this $C$-detour is external, that is, it is a $P_{i}$, which starts in $Q_{1}$ and thus must end in $Q_{2}$. We can also traverse $C^{\prime}$ in the other direction starting with $u_{\beta} u_{\beta-1}$ until we meet the $C$-detour $P^{\prime} \in\left\{P_{i-1}, P_{i+1}\right\}$ which starts in $Q_{1}$ and ends in $Q_{2}$. As $P_{i}, P^{\prime}$ do not overlap it follows that $C \cup Q \cup P_{i} \cup P^{\prime}$ contains an $M$-alternating cycle with precisely two $M$-alternating external $C$-detours, namely $P_{i}, P^{\prime}$, a contradiction.

Case 2: $u_{\alpha}^{\prime}=u_{\alpha-1}$.

Since $\operatorname{Int}(C)$ is planar and bipartite, it follows that $u_{\beta}^{\prime}=u_{\beta+1}$. We may assume that $C^{\prime}$ intersects $Q_{1}$ since otherwise we may replace $Q$ with $Q_{1}$ in $C^{\prime}$. So there must be an external $C$-detour $P_{i}: u_{b} \ldots u_{c}$ such that $\alpha<b<\beta<c$ as no external $C$-detour has both endpoints in $Q_{1}$. Since $u_{b}^{\prime} \in Q_{1}$ it follows after $P_{i}, C^{\prime}$ uses a segment of $C$ because of the minimality property of $Q$. After that segment $C^{\prime}$ uses $P^{\prime} \in\left\{P_{i-1}, P_{i+1}\right\}$ from $u_{d}$ to $u_{e}$ with $\alpha<d<\beta<e$. As $P_{i}, P^{\prime}$ do not overlap it follows that $C \cup Q \cup P_{i} \cup P^{\prime}$ contains an $M$-alternating cycle with precisely two $M$-alternating external $C$-detours, namely $P_{i}, P^{\prime}$. This contradiction proves Claim 5 .

We may assume that $P_{2}^{\prime}$ is a segment of $C$. Now $\Sigma^{\prime}=\operatorname{Int}(C) \cup \operatorname{Int}\left(C^{\prime}\right)$ is a surface with boundary, that is, $\Sigma^{\prime}$ is compact and locally homeomorphic to either a disc or a half-disc at every point. The boundary points are those points where $\Sigma^{\prime}$ is homeomorphic to a half-disc. The points of $\Sigma^{\prime}$ that are on precisely one of $C, C^{\prime}$ are clearly boundary points. So, the points on $P_{1}, P_{2}$ are all boundary points. All other boundary points are on $C$ since a point in $\operatorname{int}(C)$ is clearly not a boundary point of $\Sigma^{\prime}$. Moreover, the boundary of $\Sigma^{\prime}$ consists of simple closed curves, that is, a 2-regular subgraph of $C \cup P_{1} \cup P_{2}$. So, the boundary of $\Sigma^{\prime}$ consists of $P_{1}, P_{2}$ and two disjoint paths on $C$ connecting the four vertices $u_{2}, u_{a}, u_{b}, u_{c}$. If the boundary of $\Sigma^{\prime}$ has only one cycle, then the path on $C$ from $u_{2}$ to $u_{a}$ is contained in both $\operatorname{Int}(C)$ and $\operatorname{Int}\left(C^{\prime}\right)$. As $\operatorname{Int}\left(C^{\prime}\right)$ also contains $P_{1}$, it follows that $\operatorname{Int}\left(C^{\prime}\right)$ is nonbipartite, a contradiction. So $\Sigma^{\prime}$ has precisely two boundary cycles $R_{1}, R_{2}$, say. As they have odd length, they are not surface separating in $\Sigma$. Let $G^{\prime}$ be the part of $G$ drawn in $\Sigma^{\prime}$. As $R_{1}$ is not a face boundary of $G$ in $\Sigma$, there is an edge not in $G^{\prime}$ but incident with $R_{1}$. Let $Q$ be an $M$-alternating path starting at a vertex of $R_{1}$ such that precisely its first and last vertex are in $G^{\prime}$. If the last vertex is in $R_{1}$, then we add $Q$ to $G^{\prime}$ and call the resulting graph $G_{1}^{\prime}$. We repeat this argument with $G_{1}^{\prime}$ instead of $G^{\prime}$. Continuing like this we obtain a sequence of 
graphs $G_{1}^{\prime}, G_{2}^{\prime} \ldots$ Each $G_{i}^{\prime}$ has the property that for each vertex $x$ in $G_{i}^{\prime}$ but not in $G^{\prime}$, there is an $M$-alternating path starting with an $M$-edge incident with $x$ and terminating at $R_{1}$. As $R_{1}$ is not surface separating in $\Sigma$ we obtain in a finite number of steps an $M$-alternating path from $R_{1}$ to $R_{2}$ having only its ends in common with $G^{\prime}$. (Otherwise we would obtain a face whose boundary is not connected.) If none of the ends of $Q$ are in $C$, then (using $R_{1}, R_{2}$ ) this path $Q$ can be extended to an external $C$-detour $Q$ which is neither positive nor negative. This contradicts Claim 1 . If one or both ends of $Q$ are in $C$, we can use paths in $R_{1}, R_{2}, C$ to extend $Q$ to an $M$-alternating cycle $C^{\prime \prime}$. We claim that $C^{\prime \prime}$ is surface nonseparating in $\Sigma$ and hence noncontractible in $\Sigma$. To prove this claim we observe that $C$ has two disjoint paths joining $R_{1}, R_{2}$, and $C^{\prime \prime}$ contains precisely one of them. Now the claim follows from the following observation: $\Sigma^{\prime}$ is a surface with two holes, and the intersection of $C^{\prime \prime}$ with $\Sigma^{\prime}$ minus its boundary is a simple arc whose closure $P^{\prime \prime}$ joins the two holes. Hence $\Sigma^{\prime}$ minus $P^{\prime \prime}$ is connected, and it follows that $\Sigma^{\prime}$ has a simple closed curve crossing $C^{\prime \prime}$ precisely once which implies that $C^{\prime \prime}$ is surface nonseparating.

This contradiction completes the proof.

Corollary 17 If $G$ is an Eulerian triangulation of the torus, then $G$ has a nonbipartite spanning quadrangulation (that is, $G$ is not q-even). If, in addition, $G$ has large width, then $G$ is q-mixed.

Proof. In the torus a surface separating cycle must be contractible. Thus the first statement follows immediately from Theorem 16 and Lemma 4. However, if the width is large we know from the result of Hutchinson, Richter and Seymour [2] that $G$ is properly 4-colorable, and thus has a bipartite spanning quadrangulation.

Remark 18 After a presentation of the results in the current paper by the first author at WestFest at the Institute for Applied Mathematics in June 2014, Atsuhiro Nakamoto (private communication) and his collaborators have obtained a strengthening of Corollary 17 in that $K_{7}$ is the only q-odd triangulation of the torus. He also brought our attention to their resolution [7] of the following question relating Eulerian triangulations to spanning quadrangulations: Given a quadrangulation $Q$, how many Eulerian triangulations contain $Q$ as a spanning quadrangulation?

\section{Open problems}

We believe that the answer to Question 3 (which was first stated in an equivalent form in [4]) is negative. In other words, we conjecture:

Conjecture 19 If $G$ is a triangulation of large width on a surface, then $G$ has a bipartite spanning quadrangulation, i.e. $G$ is not q-odd.

Conjecture 20 If $G$ is a triangulation of large width on an orientable surface, then $G$ has a nonbipartite spanning quadrangulation, i.e. $G$ is not q-even.

To prove Conjecture 20, it suffices to find an alternating surface nonseparating cycle in the dual graph, by Lemma 4. We have done that for Eulerian triangulations of the torus (Corollary 17) and pointed out that this not always possible for the projective plane (Theorem 11). Conjecture 20 would follow from an affirmative answer to the following question: 
Question 21 Let $M$ be a perfect matching in a 2-connected cubic graph of large width on a fixed surface. Does $H$ contain an $M$-alternating surface nonseparating cycle?

We do not even know counterexamples of small width, in other words, is the bipartite condition necessary in Theorem 10? We believe that Theorem 16 can be extended to surface nonseparating cycles thus verifying Conjecture 20 for all Eulerian triangulations of orientable surfaces (without any width condition).

To prove Conjecture 19 by this method would amount to finding many surface nonseparating cycles as shown by Proposition 5. Thus Conjecture 19 (if true) seems much deeper than Conjecture 20 .

\section{References}

[1] J. A. Bondy and U. S. R. Murty, Graph Theory with Applications. The MacMillan Press Ltd. (1976).

[2] J. Hutchinson, R. B. Richter and P. Seymour, Coloring Eulerian triangulations. Journal of Combinatorial Theory, Series B 84 (2002), 225-239.

[3] T. Jensen and B. Toft, Graph Coloring Problems. John Wiley, New York (1995).

[4] A. Kündgen and R. Ramamurthi, Coloring face-hypergraphs of graphs on surfaces. Journal of Combinatorial Theory, Series B 85 (2002), 307-337.

[5] B. Mohar, Coloring Eulerian triangulations of the projective plane. Discrete Math. 244 (2002), 339-343.

[6] B. Mohar and C. Thomassen, Graphs on Surfaces. Johns Hopkins University Press (2001).

[7] A. Nakamoto, K. Noguchi, K. Ozeki, Extension to even triangulations, manuscript (2004).

[8] C. Thomassen, Planarity and duality of finite and infinite graphs. Journal of Combinatorial Theory, Series B 29 (1980), 244-271.

[9] C. Thomassen, Color-critical graphs on a fixed surface. Journal of Combinatorial Theory, Series B 70 (1997), 67-100. 